\title{
A Proposed Measurement of the $\beta$ Asymmetry in Neutron Decay with the Los Alamos Ultra-Cold Neutron Source
}

B.Tipton*, A. Alduschenkov, K. Asahi, T. Bowles, B. Filippone, M. Fowler, P. Geltenbort, F. Hartmann, R. Hill, A. Hime, M. Hino, S. Hoedl, G. Hogan, T. Ito, C. Jones, T. Kawai, A. Kharitonov, K. Kirch, T. Kitagaki, S. Lamoreaux, M. Lassakov, C-Y. Liu, M. Makela, J. Martin, R. McKeown, C. Morris, A. Pichlmaier, M. Pitt, Yu. Rudnev, A. Saunders, S. Seestrom, A. Serebrov, D. Smith, K. Soyama, M. Utsuro, A. Vasilev, B. Vogelaar, P. Walstrom, J. Wilhelmy, A.R. Young, J. Yuan

\author{
* Kellogg Radiation Laboratory 106-38 \\ 1200 E. California Blvd. \\ Pasadena, CA 91125 \\ E-mail: tipton@krl.caltech.edu.edu
}

\begin{abstract}
This article reviews the status of an experiment to study the neutron spinelectron angular correlation with the Los Alamos Ultra-Cold Neutron (UCN) source. The experiment will generate UCNs from a novel solid deuterium, spallation source, and polarize them in a solenoid magnetic field. The experiment spectrometer will consist of a neutron decay region in a soleniod magnetic field combined with several different detector possibilities. An electron beam and a magnetic spectrometer will provide a precise, absolute calibration for these detectors. An A-correlation measurement with a relative precision of $0.2 \%$ is expected by the end of 2002 .
\end{abstract}

\section{INTRODUCTION}

Fundamental studies of neutron beta decay provide new constraints on electroweak interactions at low energies. The leading order expression for neutron beta decay is

$$
\frac{d \sigma}{d \Omega_{e} d \Omega_{\nu}}=F\left(p_{e}\right)\left[1+\mathbf{a} \frac{\overrightarrow{p_{e}} \cdot \overrightarrow{p_{\nu}}}{E_{e} E_{\nu}}+\mathbf{A} \frac{\vec{\sigma} \cdot \overrightarrow{p_{e}}}{E_{e}}+\mathbf{B} \frac{\vec{\sigma} \cdot \overrightarrow{p_{\nu}}}{E_{\nu}}+\mathbf{D} \frac{\vec{\sigma} \cdot \overrightarrow{p_{e}} \times \overrightarrow{p_{\nu}}}{E_{\nu} E_{e}}\right]
$$

The parameters a, $\mathrm{B}$, and $\mathrm{D}$ represent the neutrino-electron correlation, the spinneutrino correlation, and the time-reversal violating correlation. The parameter $\mathrm{A}$

CP539, Symmetries in Subatomic Physics: $3^{\text {rd }}$ Int 'l. Symp., edited by X.-H. Guo, A. W. Thomas, and A. G. Williams (C) 2000 American Institute of Physics 1-56396-964-5/00/\$17.00

\section{6}


measures the parity violating correlation between the neutron spin and the electron momentum directions. The A correlation is related to the ratio of the fundamental nucleon vector and axial vector weak coupling constants, $\lambda=G_{A} / G_{V}$ through the relation,

$$
A=\frac{-2 \lambda\left(1+\lambda^{2}\right)}{1+2 \lambda^{2}} .
$$

Neutron lifetime measurements are sensitive to a different mixture of $G_{V}$ and $G_{A}$; the lifetime and A-correlation measurements may be combined to constrain these couplings separately. With the Fermi coupling constant, $G_{F}$, known from muon decay, the fundamental CKM matrix element $V_{u d}$ may be extracted. Precision determinations of $V_{u d}$ in this manner allow unitarity tests of the CKM matrix and possible access to new physics. A diagram of the current constraints on $V_{u d}$ is shown in Figure 1.

A new experiment is proposed at the Los Alamos National Laboratory to study the parity-violating spin-electron correlation in neutron beta decay. The experiment relies on a superthermal deuterium source and a spallation proton target to generate a high flux of ultra-cold neutrons (UCNs). UCNs are defined as neutrons with velocity low enough that they undergo total external reflection from the surface of materials containing them. For the case of ${ }^{58} \mathrm{Ni}$ guides, $v_{\text {neutron }}<8 \mathrm{~m} / \mathrm{s}$.

UCN from pulsed spallation source offers significant advantages over other techniques of determining the neutron beta-asymmetry. The total external reflection property allows the UCNs to be transported far from the spallation source, through significant shielding. This separates the beta spectrometers from the high background environment at the spallation target. The timing structure of the pulsed beam allows one to reject prompt beam background during pulses; the produced UCNs may be stored to deliver nearly constant UCN flux in between pulses. Finally, a several Tesla magnetic field is sufficient to overwhelm the UCN kinetic energy, allowing a solenoid magnet to select one polarization state. Nearly $100 \%$ neutron polarization is achievable.

\section{A SOLID DEUTERIUM SOURCE OF UCN}

At the heart of the proposed experiment is new type of ultra-cold neutron source using a solid deuterium $\left(\mathrm{SD}_{2}\right)$ crystal above a spallation source of cold neutrons. Figure 2 shows a schematic diagram of the proposed source. Up to $100 \mu \mathrm{C}$ of 800 $\mathrm{MeV}$ protons from LANSCE will impinge on a tungsten spallation target. Produced neutrons will reach a thin film of $\mathrm{SD}_{2}$ after being moderated in polyethylene at liquid nitrogen and liquid helium temperatures. These neutrons may phonon scatter in the $\mathrm{SD}_{2}$ crystal to come nearly to rest. Above the $\mathrm{SD}_{2}$, a structure of a ${ }^{58} \mathrm{Ni}$ coated guide surrounded by a cold beryllium reflector and a polyethylene moderator will serve both to contain the cold neutrons near the $\mathrm{SD}_{2}$ and to transport produced $\mathrm{UCN}$ away. A storage volume above the $\mathrm{SD}_{2}$ may be connected by an open valve 


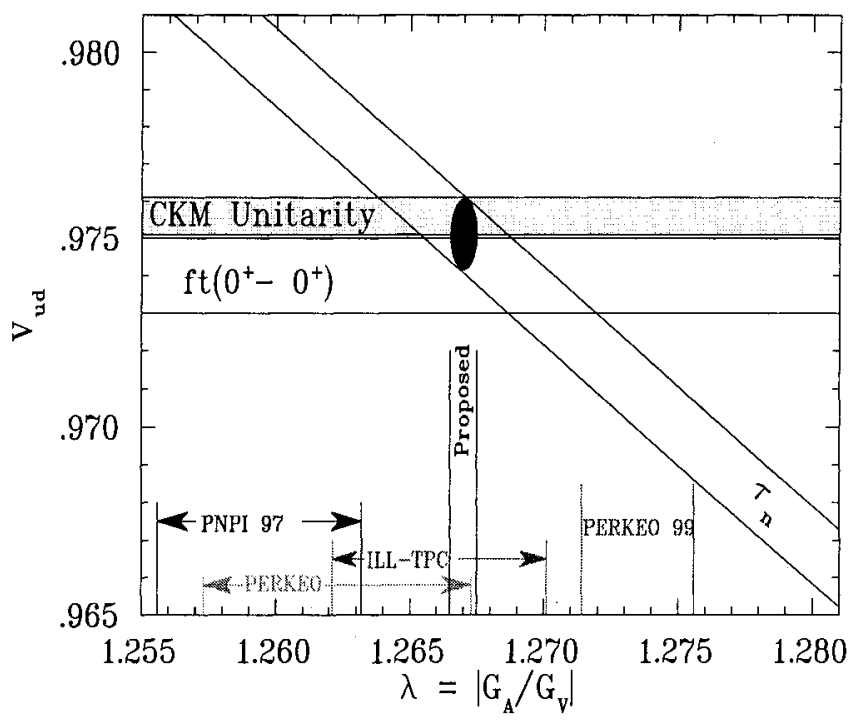

FIGURE 1. Constraints on the CKM matrix element $V_{u d}$ for recent extractions of the parameter $\lambda$ from the A-correlation. The $\lambda$ values come from PNPI 97 [2], PERKEO [1], ILL-TPC [3], and PERKEO 99 [4]. The $V_{u d}$ constraint from the ft measurement in superallowed beta decays comes from [5]. The dark oval represents the expected size of the $1 \sigma$ allowed region when the results proposed experiment are combined with the world average neutron lifetime measurement.

to the UCN source during one second proton pulses; the valve will be closed for nine seconds in between pulses while the UCN are delivered to experiments.

In runs at Los Alamos over the past year, this design was tested with a prototype UCN $\mathrm{SD}_{2}$ source connected to a ${ }^{3} \mathrm{He}$ spectrometer. The performance of the test source indicates that an equilibrium stored UCN density of $350 \mathrm{UCN} / \mathrm{cm}^{3}$ is achievable for the A-correlation experiment.

\section{THE SPECTROMETER}

A schematic design of the A-correlation experiment is shown in Figure 3. UCNs will be transported from the storage to the experiment through diamond-coated guides. On their way, they will pass through a six Tesla solenoid field, which selects one neutron spin state with near $100 \%$ efficiency. The neutrons may then be rotated periodically to the opposite spin state by subsequent passage through an adiabatic fast passage spin flipper, with expected efficiency $>99.9 \%$.

The polarized UCNs will then be fed into a $10-\mathrm{cm}$ diameter bottle inside a one Tesla superconducting solenoid. The bottle is open ended and will store the neutrons for a average of five seconds. While less than $1 \%$ of the flowing UCNs decay 


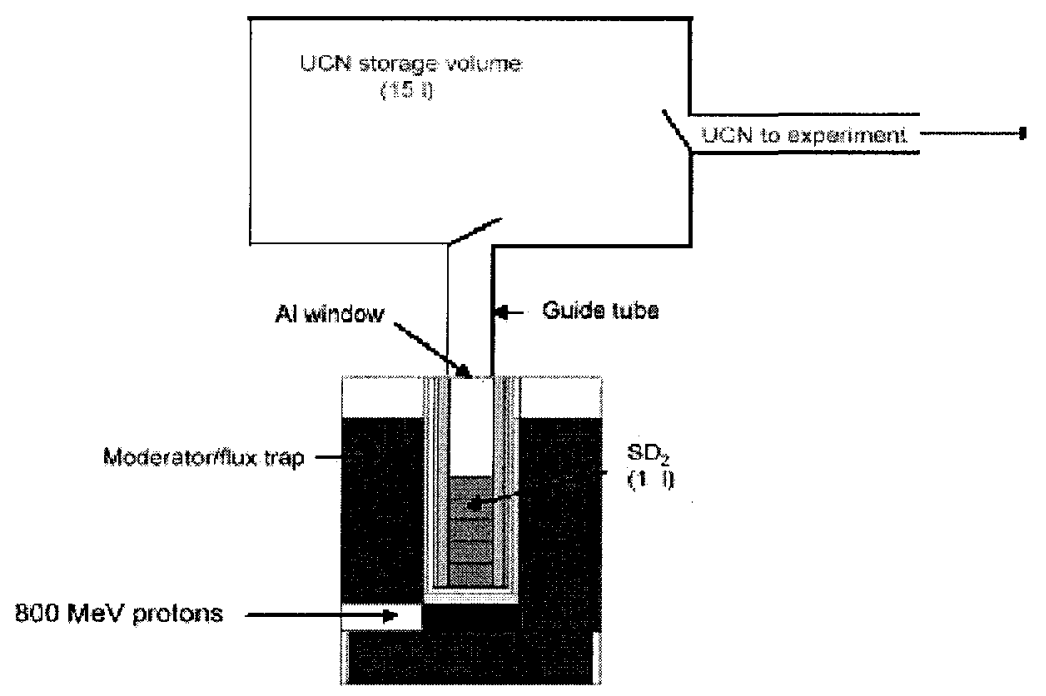

FIGURE 2. A schematic diagram of the UCN source.

inside the bottle, most are absorbed in ${ }^{6} \mathrm{Li}$ coated end chambers or flow out of the end region to neutron flux analyzing detectors.

When a UCN decays, its emitted beta will spiral in the solenoid field towards the end of the bottle; this field geometry will have nearly $4 \pi$ beta collection efficiency. Before reaching electron detectors, the betas will encounter a magnetic field expansion region, which will both orient the trajectories more perpendicular to the detector face and act as a mirror to contain the electrons in the detector region. The impact of backscattering will then be reduced.

Two electron detector designs are being developed for the experiment. In one design a 15-cm diameter Time Projection Chamber(TPC) will cover a thin scintillator. The chamber allows a precision measurement of the electron trajectory for better fiducial volume definition and statistical sensitivity. The requirement of a TPC-scintillator coincidence in each event also eliminates backgrounds, such as from cosmic rays. Alternatively, a double-sided silicon strip detector may be placed in the experiment to provide excellent energy resolution. The combination of both detector technologies with differing sensitivities and systematics will allow cross-checks in the experiment.

A total of $108 \mathrm{~Hz}$ of neutrons will decay in the experiment, with $87 \mathrm{~Hz}$ analyzable after efficiencies and cuts are considered. Backgrounds in the experiment are essentially negligible. With the experiment comissioning by the end of 2001 the anisotropy in neutron beta decay is then expected to be measured to the $0.16 \%$ level statistically by the end of 2002 .

The anticipated largest source of systematic uncertainty comes from knowledge 


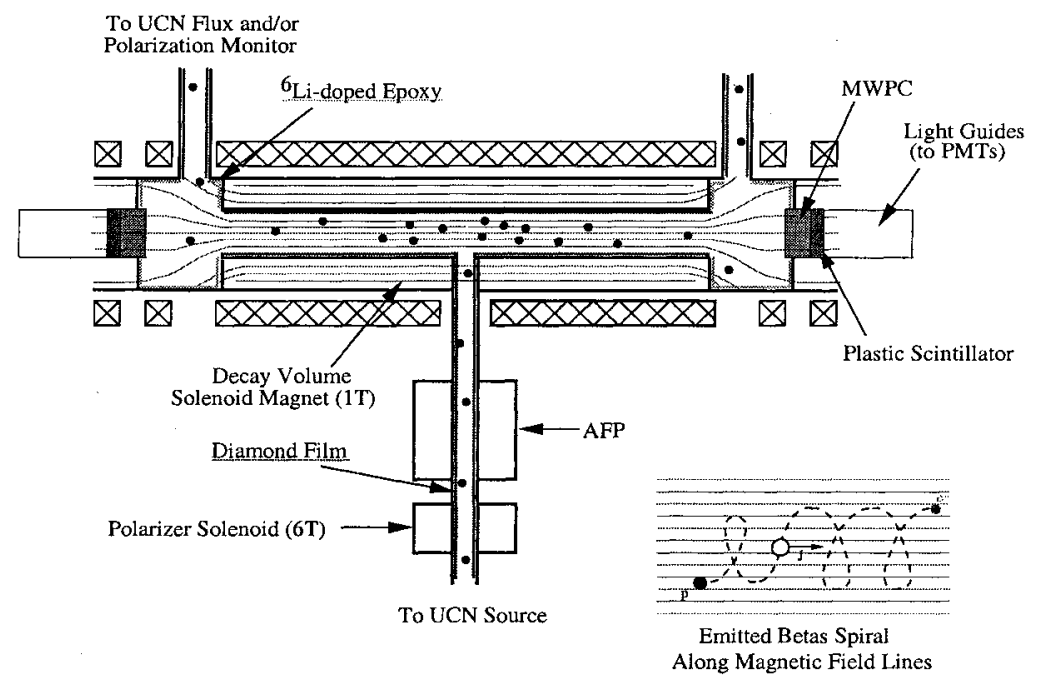

FIGURE 3. An overhead view of the spectrometer for the A-correlation experiment.

of the detector performances. The experimental goal is to keep the size of this systematic below $0.03 \%$ for a total systematic uncertainty of $0.042 \%$.

An ambitious program has been begun to allow detailed study of the electron detectors after they are constructed. A combination of a custom built $150 \mathrm{keV}$ electron gun and the $1 \mathrm{MeV}$ Dynamotron at NASA's Jet Propulsion Laboratory provide continuous, stable, tunable electron beam from $5 \mathrm{keV}$ to $1 \mathrm{MeV}$. A double focusing magnetic spectrometer has been constructed at Caltech to calibrate the electron beam energy to better than $0.3 \%$. The combination of these devices will allow an extremely high precision characterization of the detector efficiency, calibration, linearity, resolution, and backscattering probability before and during the experiment.

\section{CONCLUSION}

A new experiment is proposed at Los Alamos to provide the world's best measurement of the A-correlation in neutron beta decay. The experimental collaboration plans to measure $\mathrm{A}$ to a precision of $0.2 \%$ by then end of 2002 . This is an improvement of over a factor of four compared to the most recent A-correlation experiment [4]. The black dot in Figure 1 shows the impact of this measurement on the determination of $V_{u d}$, when combined with world data on the neutron lifetime. 


\section{ACKNOWLEDGMENTS}

The author would like to acknowledge the support of the U.S. National Science Foundation.

\section{REFERENCES}

1. Bopp, P et. al., Phys. Rev. Lett. 56, 919 (1986).

2. Erozoliminskii, B. G.et. al., Phys. Lett. B236, 33 (1991).

3. Schreckenbach, K. et. al., Phys. Lett. B349, 427 (1995).

4. Reich, J. et. al., Phys. Lett. 440, 535 (2000).

5. Hardy, J.C., Towner, I.S., nucl-th/9807049. 